\title{
A relation between the resonance neutron peak and ARPES data in cuprates
}

\author{
Ar. Abanov and Andrey V. Chubukov \\ Department of Physics, University of Wisconsin, Madison, WI 53706
}

(September 13, 2018)

We argue that the resonant peak observed in neutron scattering experiments on superconducting cuprates and the peak/dip/hump features observed in ARPES measurements are byproducts of the same physical phenomenon. We argue that both are due to feedback effects on the damping of spin fluctuations in a $d$-wave superconductor. We consider the spin-fermion model at strong coupling, solve a set of coupled integral equations for fermionic and bosonic propagators and show that the dynamical spin susceptibility below $T_{c}$ possesses the resonance peak at $\Omega_{r e s} \propto \xi^{-1}$. The scattering of these magnetic excitations by electrons gives rise to a peak/dip/hump behavior of the electronic spectral function, the peak-dip separation is exactly $\Omega_{r e s}$.

PACS numbers:71.10.Ca,74.20.Fg,74.25.-q

One of the most intriguing recent developments in the physics of high $T_{c}$ materials is the realization that not only the normal but also the superconducting state of cuprates is not described by a weak coupling theory. In particular, ARPES experiments on Bi2212 have demonstrated [1.2] that even in slightly overdoped cuprates at $T \ll T_{c}$, the spectral function $A(\mathbf{k}, \omega)$ near $(0, \pi)$ does not possess a single quasiparticle peak at $\omega=\sqrt{\Delta_{\mathbf{k}}^{2}+\epsilon_{\mathbf{k}}^{2}}$, where $\Delta_{\mathbf{k}}$ is the superconducting gap and $\epsilon_{\mathbf{k}}$ is the fermionic dispersion. Instead, it displays a sharp peak which virtually does not disperse with $k$, a dip at frequencies right above the peak, and then a broad maximum (hump) which disperses with $k$ and gradually recovers the normal state dispersion [1]. Simultaneously, the neutron scattering experiments on near optimally doped $Y B C O$ [3] and Bi2212 [4] at $T \ll T_{c}$ have detected a sharp resonance peak in the dynamical structure factor $S(q, \Omega) \propto \chi^{\prime \prime}(q, \Omega)$ centered at $q=Q=(\pi, \pi)$ and at frequencies $\sim 40 \mathrm{meV}$.

In this communication we show that the resonance peak in $S(Q, \Omega)$ and the peak/dip/hump features in $A(k, \omega)$ can be explained simultaneously by strong interaction between electrons and their collective spin degrees of freedom which near the antiferromagnetic instability are peaked at or near $Q$. Specifically, we demonstrate that a $d$-wave superconductor possesses propagating collective spin excitations at frequencies smaller than twice the maximum value of the $d$-wave gap. The propagating spin modes give rise to a sharp peak in $S(Q, \Omega)$ at $\Omega=\Omega_{\text {res }} \propto \xi^{-1}$ where $\xi$ is the spin correlation length. The interaction with collective spin excitations yields the fermionic self-energy $\Sigma_{\omega}$ which at $T=0$ has no imaginary part up to a frequency $\omega_{0}$ which exceeds the measured superconducting gap by exactly $\Omega_{\text {res }}$.

The point of departure for our analysis is the spinfermion model for cuprates which is argued [6] to be the low-energy theory for Hubbard-type lattice fermion models. The model is described by

$$
\begin{aligned}
\mathcal{H}= & \sum_{\mathbf{k}, \alpha} \mathbf{v}_{\mathbf{F}}\left(\mathbf{k}-\mathbf{k}_{F}\right) c_{\mathbf{k}, \alpha}^{\dagger} c_{\mathbf{k}, \alpha}+\sum_{q} \chi_{0}^{-1}(\mathbf{q}) \mathbf{S}_{\mathbf{q}} \mathbf{S}_{-\mathbf{q}}+ \\
& g \sum_{\mathbf{q}, \mathbf{k}, \alpha, \beta} c_{\mathbf{k}+\mathbf{q}, \alpha}^{\dagger} \sigma_{\alpha, \beta} c_{\mathbf{k}, \beta} \cdot \mathbf{S}_{-\mathbf{q}} .
\end{aligned}
$$

Here $c_{\mathbf{k}, \alpha}^{\dagger}$ is the fermionic creation operator for an electron with crystal momentum $\mathbf{k}$ and spin $\alpha, \sigma_{i}$ are the Pauli matrices, and $g$ is the coupling constant which measures the strength of the interaction between fermions and the collective bosonic spin degrees of freedom. The latter are described by $\mathbf{S}_{\mathbf{q}}$ and are characterized by a bare spin susceptibility $\chi_{0}(\mathbf{q})=\chi_{0} \xi^{2} /\left(1+(q-Q)^{2} \xi^{2}\right)$.

Eq. (1) gives rise to fermionic and bosonic self-energies and is particularly relevant for fermions near hot spots - the points at the Fermi surface separated by $Q$. In cuprates, the hot spots are located near $(0, \pi)$ and symmetry related points. The presence of hot spots is essential for our consideration because the fermions near these points are mostly affected by the interaction with antiferromagnetic spin fluctuations, and at the same time, they produce the dynamical part of the spin propagator because a spin fluctuation with a momentum near $Q$ can only decay into fermions near hot spots.

The normal state properties of the spin-fermion model have recently been analysed and compared with the experiments [6]7]. It was argued that the experimental situation in cuprates corresponds to a strong coupling limit $R=\bar{g} / v_{F} \xi^{-1} \gg 1$, where $\bar{g}=g^{2} \chi_{0}$ is the measurable effective coupling constant. The clearest experimental indication for this is the absence of the sharp quasiparticle peak in the normal state ARPES data for optimally doped and and underdoped cuprates [1,2]. At strong coupling, a conventional perturbation theory does not work, but it turns out that one can single out the most divergent diagrams, and incorporate them into a new "meanfield" ground state. This "mean-field" theory becomes exact if one formally sets the number of hot spots $N=8$ to infinity. At finite $N$, the expansion around the new vacuum holds in in $(1 / N) \log R$, but the prefactors are very small [6] such that for practical purposes, one can restrict with the $N=\infty$ theory except very near the antiferromagnetic transition. 
This $N=\infty$ theory has some similarities with the mean-field $d=\infty$ theories [8]: it incorporates the dominant $(\sim R)$ self-energy correction which depends only on frequency, and also includes the dominant bosonic selfenergy which is the spin polarization bubble made of the renormalized fermions. The corresponding set of selfconsistent equations is presented in Eq. (2) for a superconducting state. The normal state results are obtained by setting $\Delta=0$.

The key physical effect which the $N=\infty$ theory describes is the progressive desctruction, with increasing $R$, of the coherent quasiparticle peak. At the same time, the fermionic incoherence has no feedback on spin susceptibility which still has a simple relaxational form: $\chi^{-1}(q, \Omega)=\chi_{0} \xi^{2} /\left(1+(q-Q)^{2} \xi^{2}-i \Omega / \omega_{s f}\right)$ where $\omega_{s f}=(2 \pi / N)\left(v_{F} \xi^{-1}\right) / R$ [6]. The absence of the feedback effect on spins is a quite general consequence of the fact that fermionic self-energy, abeit strong, has no dependence on the quasiparticle momentum [9].

In the superconducting state, this argument does not apply any more because superconducting and normal state Green's functions have different momentum dependences As a result, the feedback effect on spins is present, and already at the $N=\infty$ level one has to solve a set of coupled integral equations for the fermionic propagator and the spin polarization operator. This is the key intent of the present work. We however will not attempt to self-consistently find also the pairing susceptibility which in the spin-fermion model results from multiple spin-fluctuation exchanges in the particle-particle channel [10]. Instead, we assume that below $T_{c}$ the pairing susceptibility is a conventional $\delta$-function of a total momentum and frequency of a pair with the $d$-wave amplitude $\Delta_{k}^{2}$. In other words, we will not distinguish between the true superconducting gap and the pseudogap. The full consideration should indeed include pairing fluctuations into the self-consistent procedure. We will also neglect the processes which scatter fermions near $(0, \pi)$ into fermions with momenta along zone diagonal where the $d$-wave gap is absent. The contributions from these processes soften sharp features associated with the $k$-independent gap, but are likely to be small numerically as they involve high energy spin fluctuations with momenta far from $Q$. Still, however, we will fully explore the fact that for $d_{x^{2}-y^{2}}$ symmetry of the gap, $\Delta_{k+Q}=-\Delta_{k}$.

A simple experimentation shows that for a $\delta$-functional pairing susceptibility, the $N=\infty$ theory in the superconducting state has the same selfconsistent structure as in the normal state but operates with normal and anomalous fermionic Green's functions $G(k, \omega)=G_{0}(k, \omega) /\left(1+\Delta_{k}^{2} G_{0}(k, \omega) G_{0}(-k,-\omega)\right)$ and $F(k, \omega)=-i \Delta_{k} /\left(1+\Delta_{k}^{2} G_{0}(k, \omega) G_{0}(-k,-\omega)\right)$ where $G_{0}^{-1}(k, \omega)=\omega-\Sigma_{\omega}-\mathbf{v}_{F}\left(\mathbf{k}-\mathbf{k}_{F}\right)$ is the zero-order Green's function for $1 / N$ expansion. This $G_{0}(k, \omega)$ contains a self-energy which results from an exchange of a spin fluc- tuation with $\chi(q, \Omega)=\chi_{0} \xi^{2} /\left(1+(q-Q)^{2} \xi^{2}-\Pi_{\Omega}\right)$ where the spin polarization bubble $\Pi_{\Omega}$ is by itself a convolution of $G G$ and $F F$. This construction yields a set of two coupled integral equations

$$
\begin{aligned}
\Sigma_{\omega}= & 3 i g^{2} \int \frac{d^{2} q d \Omega}{(2 \pi)^{3}} G(k+q, \omega+\Omega) \chi(q, \Omega) \\
\Pi_{\Omega}= & -2 N i \bar{g} \xi^{2} \int \frac{d^{2} k d \omega}{(2 \pi)^{3}}(G(k, \omega) G(k+Q, \omega+\Omega) \\
& +F(k, \omega) F(k+Q, \omega))
\end{aligned}
$$

These equations has to be solved to leading order in $1 / N$ (this, e.g., eliminates $k$-dependence in $\Sigma$ ). Higer order self-energy and vertex correcions are small in $1 / N$ and we neglect them.

It is instructive to consider first the solution of Eqs (2) in the weak coupling limit $\Delta \ll \bar{g} \ll v_{F} \xi^{-1}$. To first approximation, $\Pi_{\Omega}$ can then be evaluated with the free fermion Green's functions. This has been done before 11] and we just quote the result: in the superconducting state, $\Pi_{\Omega}$ has both real and imaginary parts. The $\operatorname{Im} \Pi_{\Omega}=0$ for $\Omega<2 \Delta$, it jumps at $\Omega=2 \Delta$ to $\pi \Delta / \omega_{s f}$, and then increases and approaches $\Omega / \omega_{s f}$ at $\Omega \gg 2 \Delta$. This behavior is similar to that in an $s$-wave superconductor except for the jump which is absent in $s$-wave case and is directly related to the fact that $\Delta_{k+Q}=-\Delta_{k}$.

The $\operatorname{Re} \Pi_{\Omega}$ can be obtained either directly or using the Kramers-Kronig relation. At $\Omega \ll \Delta$, we have $\operatorname{Re} \Pi(\omega)=(\pi / 8) \Omega^{2} /\left(\Delta \omega_{s f}\right)$. It diverges at $2 \Delta$ as $\Pi_{\Omega}=\left(\Delta / \omega_{s f}\right) \log (2 \Delta /|2 \Delta-\Omega|)$ because of the jump in $\operatorname{Im} \Pi_{\Omega}$, and decreases at larger frequencies. Due to the divergence, $R e \Pi_{\Omega}$ reaches 1 at a frequency $\Omega_{\text {res }}$ which is less than $2 \Delta$, i.e., when $\operatorname{Im} \Pi_{\Omega}$ is still zero. Explicitly, $\Omega_{\text {res }}=2 \Delta(1-Z)$ where $Z \propto e^{-\omega_{s f} /(2 \Delta)}$. Near $\Omega_{\text {res }}$, $\chi(Q, \Omega) \propto Z /\left(\Omega-\Omega_{\text {res }}-i \delta\right)$, i.e., the dynamical structure factor has a resonance peak.

Consider next the fermionic spectral function. Without self-energy corrections, $A(k, \omega)$ near a Fermi surface resonates at $\omega_{\text {res }}=\Delta$. The self-energy gives rise to a fermionic decay. For an $s$-wave superconductor, the onset frequency for a decay is $3 \Delta$, and $\operatorname{Im} \Sigma(k, \omega)$ emerges as $(\omega-3 \Delta)^{1 / 2}[5]$. The presence of the resonance mode qualitatively changes this picture because a fermion can decay into this mode starting from $\omega_{0}<3 \Delta$. A simple power counting shows that this process yields a finite jump of $\operatorname{Im} \Sigma_{\omega}$ at the onset frequency and hence the logarithmical singularity in $\operatorname{Re} \Sigma_{\omega}$. The latter in turn increases the self-energy at $\omega \sim \Delta$ and shifts downwards $\omega_{\text {res }}$ (which is the measured gap), and the onset frequency for $\operatorname{Im} \Pi_{\Omega}$ which, as one can easily demonstrate, exactly equals $2 \omega_{\text {res }}$. The amounts of the shifts and the amplitude of the jump in $\operatorname{Im} \Sigma_{\omega}$ can be obtained explicitly from Eqs. (2). We found $\omega_{0}=3 \Delta(1-\epsilon)$, $\omega_{\text {res }}=\Delta(1-\epsilon)$, and $\delta\left(\operatorname{Im} \Sigma_{\omega_{0}}\right)=(\pi \Delta / \log 2) \epsilon$, where $\epsilon=\left(3 \log 2 \sqrt{N} /(64 \sqrt{\pi})(\bar{g} / \Delta)^{1 / 2} e^{-\omega_{s f} /(2 \Delta)}\right.$. 
We see that the $d$-wave form of the gap yields qualitative changes in the system behavior compared to the $s$-wave case, but at small coupling these changes are exponentially small and can hardly be measured. In particular, the resonance peak in $S(Q, \omega)$ should be smeared out already by a small experimental resolution. The weak coupling results are shown in Fig 1 .
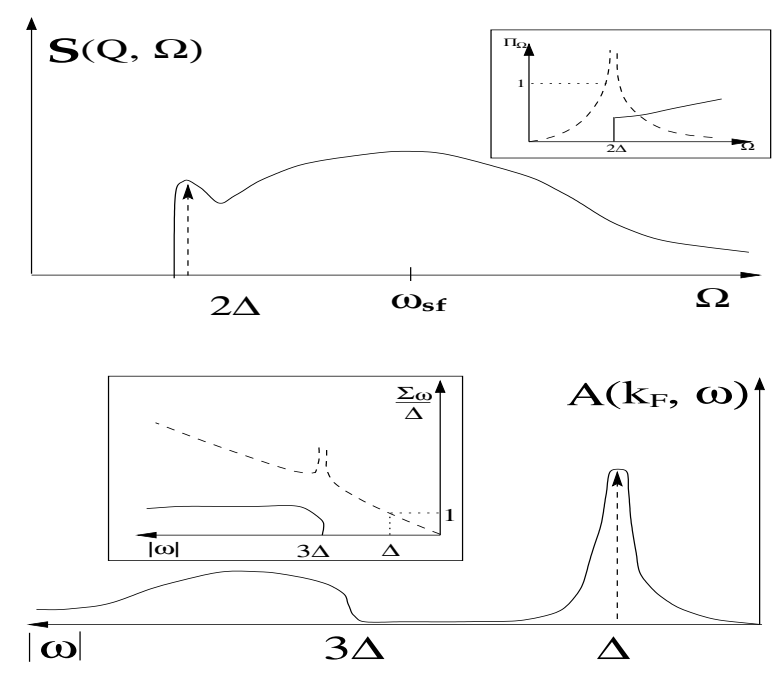

FIG. 1. The $T=0$ weak coupling behavior of the dynamical structure factor and the spectral function. Solid lines are schematic solutions of Eqs. (1) broadened by experimental resolution. Without resolution, the peaks are $\delta$-functions as indicated by arrows. The insets show the spin polarization operator $\Pi_{\Omega}$ and the fermionic self-energy $\Sigma_{\omega}$ (solid lines - imaginary parts, dashed lines - real parts). The vertical dashed lines denote logarithmical singularities.

We now turn to strong coupling limit $\bar{g} \gg \Delta \gg$ $\left(\omega_{s f} \bar{g}\right)^{1 / 2}$. Solving Eq. (2) as explained below, we found that the peak frequency in $A\left(k_{F}, \omega\right)$ (i.e., the measured gap) is now $\omega_{\text {res }}=\bar{\Delta} \sim \Delta^{2} / \bar{g}$. The nonzero $\operatorname{Im} \Pi_{\Omega}$ and $\operatorname{Im} \Sigma_{\omega}$ appear respectively at $2 \bar{\Delta}$ and $\omega_{0}=\bar{\Delta}(1+a)$ where $a \propto\left(\omega_{s f} / \bar{\Delta}\right)^{1 / 2} \propto \xi^{-1}$. The amounts of jumps in $\operatorname{Im} \Pi_{\Omega}$ and $\operatorname{Im} \Sigma_{\omega}$ both scale as $a^{1 / 2}$ and completely disappear at $\xi=\infty$ when $\omega_{s f}=0$. Above the threshold, $\operatorname{Im} \Sigma_{\omega}$ first increases as $\operatorname{Im} \Sigma_{\omega} \propto\left(\omega-\omega_{0}\right)^{\nu}$ where $\nu=\sqrt{3}-1$, and then recovers the normal state, $\sqrt{\omega}$ behavior. Substituting this $\Sigma$ into $G(\omega)$, we found that it possesses a peak at $\omega_{\text {res }}$, a dip at $\omega_{0}$ and a hump at $\omega_{\text {hump }}=\omega_{\text {res }}(1+b)$ where $b \sim(\bar{g} / \bar{\Delta})^{3 /(4 \nu)} R^{1 /(2 \nu)} \log R \propto \xi^{-1 /(2 \nu)} \log \xi$. At $\xi=\infty$, peak, dip and hump positions coincide with each other, and the peak/dip/hump structures transformes into the edge singularty: $A(\omega) \propto\left(\omega-\omega_{\text {res }}\right)^{-\nu}$.

Further, the fact that $\operatorname{Im} \Pi_{\Omega}=0$ up to $2 \omega_{\text {res }}$ implies, via Kramers-Kronig relation, that at small frequencies $R e \Pi_{\Omega} \propto \Omega^{2} /\left(\omega_{s f} \bar{\Delta}\right)$. Substituting this result into $S(q, \omega)$, we find that it possesses a resonance peak at $\Omega_{\text {res }} \sim\left(\omega_{s f} \bar{\Delta}\right)^{1 / 2} \sim \xi^{-1} \ll 2 \bar{\Delta}$. At $q \neq Q$, the peak disperses with $q$ as $\Omega^{2}=\omega_{\text {res }}^{2}\left(\left(1+((q-Q) \xi)^{2}\right.\right.$, just as a conventional spin wave, untill $\Omega$ reaches $2 \omega_{\text {res }}$, and at larger frequencies disappears due to damping.
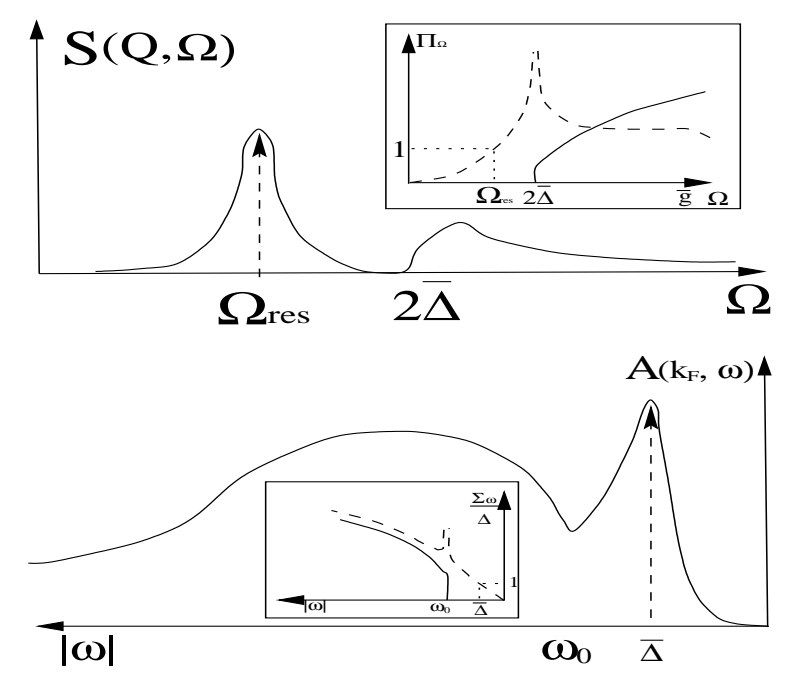

FIG. 2. Same as in Fig 1 but at strong coupling. The resonance and onset frequencies are presented in the text. The spin resonance frequency $\Omega_{\text {res }} \propto \xi^{-1}$, is equal to the distance between the measured gap $\bar{\Delta}$ and the dip frequency $\omega_{0}$. The hump frequency differes from $\bar{\Delta}$ roughly by $\xi^{0.7}$.

The strong-coupling behavior of $S(Q, \Omega)$ and $A(k, \omega)$ is presented in Fig. 2. We see that (i) $S(q, \omega)$ possesses a sharp resonance peak at $\Omega_{\text {res }} \sim \xi^{-1}$ which shifts with underdoping to lower frequencies, and (ii) $A\left(k_{F}, \omega\right)$ possesses a quasiparticle peak at $\omega=\omega_{\text {res }}$, a dip at $\omega_{0}=\omega_{\text {res }}+\Omega_{\text {res }}$, where $\operatorname{Im} \Sigma_{\omega}$ first appears, and a broad maxumum at a somewhat higher frequency $\omega_{\text {hump }}$. As the momentum moves away from the Fermi surface, the spectral function for frequencies larger than $\omega_{0}$ disperses with $k$ and recovers the normal state, non-Fermi liquid form with a broad maximum at $\omega \sim \epsilon_{k}^{2} / \bar{g}$. The quasiparticle peak however cannot move further than $\omega_{0}$ because of a strong fermionic damping above threshold. We found that it gets pinned at $\omega_{0}$ and just gradually looses its strength with increasing $k-k_{F}$.

We emphasize that although the resonance frequency in $S(Q, \omega)$ continuously evolves from weak to strong coupling, the physics changes qualitatively between the two limits. At weak coupling, the peak is solely due to a jump in $\operatorname{Im} \Pi_{\Omega}$. At strong coupling, the jump is almost gone, and the existence of peak is due to $\Omega^{2}$ behavior of $R e \Pi_{\Omega}$ which is related to vanishing $\operatorname{Im} \Pi_{\Omega}$ below $2 \omega_{\text {res }}$.

We now briefly discuss how we obtained these results. We first integrated over momentum in (2) and for $R=$ $\bar{g} /\left(v_{F} \xi^{-1}\right) \gg 1$ obtained

$$
\begin{gathered}
\Sigma_{\omega}=\frac{3 R}{8 \pi} \int d \Omega \frac{\Sigma_{\omega+\Omega}}{\sqrt{\Sigma_{\omega+\Omega}^{2}-\Delta^{2}}} \frac{1}{\sqrt{1-\Pi_{\Omega}}} \\
\Pi_{\Omega}=\frac{i}{2} \int \frac{d \omega}{\omega_{s f}}\left(\frac{\Sigma_{\Omega-\omega} \Sigma_{\omega}+\Delta^{2}}{\sqrt{\sum_{\Omega-\omega}^{2}-\Delta^{2}} \sqrt{\Sigma_{\omega}^{2}-\Delta^{2}}}+1\right)
\end{gathered}
$$


We then performed self-consistent calculations: we first obtained regular terms in $R e \Sigma$ and $R e \Pi$ by expanding (3, 4) in the external frequencies. We obtained $\operatorname{Re} \Sigma_{\omega} \propto \omega(\bar{g} / \bar{\Delta})^{1 / 2}$ and $\operatorname{Re} \Pi_{\Omega} \propto \Omega^{2} /\left(\bar{\Delta} \omega_{s f}\right)$. In both cases, the integrals are confined to frequencies $\sim \bar{\Delta}$ where the system interpolates between normal state and superconducting behavior, and for estimates, one can use in the integrands the known normal state results for $\Sigma_{\omega}$ and $\Pi_{\omega}$. We then assumed that at some finite frequency $\omega_{0}$ $\operatorname{Im} \Sigma_{\omega}$ jumps from 0 to some finite value, considered the onset frequency and the amount of the jump as input parameters, and used Kramers-Kronig relation to calculate the logarithmicaly singular term in $R e \Sigma_{\omega}$. Adding it to a regular $\operatorname{Re} \Sigma_{\omega} \propto \omega$, we find $\omega_{\text {res }}$ where $\omega+\operatorname{Re} \Sigma_{\omega_{\text {res }}}=\Delta$. Substituting next $\operatorname{Re} \Sigma_{\omega}$ into (4) and using the spectral representation for $I m \Pi_{\Omega}$, we find the threshold frequency for $I m \Pi_{\Omega}$ at $2 \Omega_{\text {res }}$ and the amount of the jump at the threshold. We then again use Kramers-Kronig relation to calculate a logarithmically singular contribution to $R e \Pi$, add it to a regular $R e \Pi_{\Omega} \propto \Omega^{2}$, and subsitute the result into (3) for $\Sigma_{\omega}$. Using again the spectral representation for $\operatorname{Im} \Sigma_{\omega}$, we find two self-consistent equations for threshold frequency $\omega_{0}$ and for the amount of the jump at the threshold. These calculations are not exact because we only know regular $\operatorname{Re} \Sigma_{\omega}$ and $R e \Pi_{\Omega}$ upto overall numerical factors, but they yield correct functional forms of the resonance and threshold frequencies.

We now compare our results with the data. Qualitatively, the peak/dip/hump behavior of $A(k, \omega)$, the absence of the peak dispersion with $k$, and the presence of the dispersing resonance mode in $S(q, \omega)$ all agree with the ARPES and neutron measurements in $Y B C O$ and Bi2212 迎田. More quantitatively, we predict that the peak-dip separation in $A(k, \omega)$ at a hot spot exactly equals to the resonance frequency in $S(Q, \Omega)$. Experimentally, in near optimally doped, $T_{c}=87 \mathrm{~K} B i 2212$, $\omega_{0}-\omega_{r e s} \approx 42 \mathrm{meV}$ [1]. Recent neutron scattering data 44 on Bi2212 with nearly the same $T_{c}=91 \mathrm{~K}$ yielded $\Omega_{r e s}=43 \mathrm{meV}$, in full agreement with the theory. We also predict that the peak, dip and hump frequencies should tend to the same value as $\xi \rightarrow \infty$. At large but finite $\xi$, we predict that $\left(\omega_{\text {hump }} / \omega_{\text {res }}-1\right) \propto\left(\omega_{0} / \omega_{\text {res }}-1\right)^{\beta}$ where $\beta=(\sqrt{3}+1) / 4 \sim 0.7$.

We now connect our work to earlier studies. That the interaction with a nearly resonant collective mode peaked at $Q$ explains the ARPES data has been known for some time, and qualitative arguments have been displayed first in 12] and then in [1]. Ref [1] also conjectured that the peak-dip separation may be related to a neutron peak frequency. It has been also realized earlier that in a $d$ - wave superconducting Fermi gas, $S(Q, \Omega)$ contains a resonance peak exponentially close to $2 \Delta$ [11. From this perspective, the key intension of this work was to present the quantitative results for cuprates by performing actual strong coupling calculations, and to explicitly relate ARPES and neutron scattering data.
Morr and Pines [13] obtained the spin-wave like dispersion in $\chi(q, \Omega)$ below $T_{c}$ by phenomenologically adding the $\Omega^{2}$ term to the bare susceptibility. This term should be in the form $\Omega^{2} / \epsilon_{F}$ as it can only come from fermions located far away from the Fermi surface. We have demonstrated that at $T<T_{c}$, the spin-fermion model of Eq (1) by itself generates an $\Omega^{2} / \Delta$ term which for $\Delta \ll \epsilon_{F}$ completely overshadows a possible bare $\Omega^{2}$ term.

Morr and one of us [7] considered an approximate solution of Eq. (4) assuming that $\Pi_{\Omega}$ still has the same purely relaxational form $i \Omega / \omega_{s f}$ as in the normal state, but $\omega_{s f}$ is momentum dependent. Comparing our results with [7], we found that the approximate solution for $A(k, \omega)$ captures the main features of the full solution, i.e., the peak/dip/hump structure of $A(k, \omega)$, but yields incorrect values of the peak and dip frequencies for $\bar{\Delta} \gg \omega_{s f}$.

J. Brinckman and P. Lee studied the evolution of the resonance peak within the slave boson theory [14]. Their philosophy and the results are similar to ours.

To summarize, we considered the superconducting phase of cuprates and demonstrated that the resonance peak in the dynamic structure factor and the peak/dip/hump structure of the electronic spectral function near $(0, \pi)$ can simultaneously be explained by a strong spin-fermion interaction. The peak-dip separation at a hot spot exactly equals to the resonance neutron frequency and vanishes at $\xi=\infty$. The peak-hump separation also vanishes, but with a smaller power of $\xi^{-1}$.

It is our pleasure to thank G. Blumberg, A. Finkelstein, R. Joynt, G. Kotliar, A. Millis, D. Morr, M. Norman, D. Pines and J. Schmalian for useful conversations. The research was supported by NSF DMR-9629839.

[1] M. R. Norman et al., Phys. Rev. Lett. 79, 3506 (1997).

[2] Z-X. Shen et al, Science 280, 259 (1998).

[3] H.F. Fong et al, Phys. Rev. B 54, 6708 (1996); P. Dai et al, Science 284, 1344 (1999).

[4] H.F. Fong et al, Nature 398, 588 (1999).

[5] P.B. Littlewood and C.M. Varma, Phys. Rev. B 46, 405 (1992).

[6] A. Chubukov, Europhys. Lett. 44, 655 (1997).

[7] A. Chubukov and D. Morr, Phys. Rev. Lett. 81, 4716 (1998).

[8] A. Georges et al, Rev. Mod. Phys., 68, 13 (1996).

[9] L. Kadanoff, Phys. Rev. 132, 2073 (1963).

[10] P. Monthoux and D. Pines, Phys. Rev. B 47, 6069 (1993) D.J. Scalapino, Phys. Rep. 250, 329 (1995).

[11] D.Z. Liu, Y. Zha and K. Levin, Phys. Rev. Lett. 75, 4130 (1995); I. Mazin and V. Yakovenko, ibid 75, 4134 (1995); (1991); A. Millis and H. Monien, Phys. Rev. B 54, 16172 (1996); N. Bulut and D. Scalapino, Phys. Rev. B 53, 5149 (1996). 
[12] Z-X. Shen and J.R. Schrieffer, Phys. Rev. Lett. 78, 1771 (1997).

[13] D.K. Morr and D. Pines, Phys. Rev. Lett. 81, 1086 (1998).

[14] J. Brinckmann and P.A. Lee, Phys. Rev. Lett. 82, 2915 (1999). 